\title{
Combination of Tramadol with Minocycline Exerted Synergistic Effects on a Rat Model of Nerve Injury-Induced Neuropathic Pain
}

\author{
Xiao-Peng Mei ${ }^{a, b}$ Lei Chen ${ }^{b}$ Wei Wang ${ }^{a, b}$ Dan Wu ${ }^{a} \quad$ Li-Ying Wang $^{b}$ \\ Ting Zhang $^{\text {b Hui Zhang }}{ }^{\mathrm{a}}$ Li-Xian Xu ${ }^{\mathrm{a}}$ Yun-Qing Lib \\ ${ }^{a}$ Department of Anesthesiology, School of Stomatology, and ${ }^{b}$ Department of Anatomy, Histology and Embryology, \\ K.K. Leung Brain Research Centre, Fourth Military Medical University, Xi'an, PR China
}

\section{Key Words}

Microglia · Spinal nerve ligation • Antiallodynia • Analgesic • Spinal cord

\begin{abstract}
Neuropathic pain is a refractory clinical problem. Certain drugs, such as tramadol, proved useful for the treatment of neuropathic pain by inhibiting the activity of nociceptive neurons. Moreover, studies indicated that suppression or modulation of glial activation could prevent or reverse neuropathic pain, for example with the microglia inhibitor minocycline. However, few present clinical therapeutics focused on both neuronal and glial participation when treating neuropathic pain. Therefore, the present study hypothesized that combination of tramadol with minocycline as neuronal and glial activation inhibitor may exert some synergistic effects on spinal nerve ligation (SNL)-induced neuropathic pain. Intrathecal tramadol or minocycline relieved SNL-induced mechanical allodynia in a dose-dependent manner. SNL-induced spinal dorsal horn Fos or OX42 expression was downregulated by intrathecal tramadol or minocycline. Combination of tramadol with minocycline exerted powerful and synergistic effects on SNL-induced neuropathic pain also in a dose-dependent manner. Moreover, the drug com-
\end{abstract}

\begin{tabular}{|c|c|}
\hline KARGER & $\begin{array}{ll}\text { (c) } 2012 \text { S. Karger AG, Basel } & \text { Karger } \\
1424-862 X / 13 / 0214-0184 \$ 38.00 / 0 & \text { Open access }\end{array}$ \\
\hline $\begin{array}{l}\text { E-Mail karger@karger.com } \\
\text { www.karger.com/nsg }\end{array}$ & $\begin{array}{l}\text { This is an Open Access article licensed under the terms of the } \\
\text { Creative Commons Attribution-NonCommercial 3.0 Un- } \\
\text { ported license (CC BY-NC) (www.karger.com/OA-license), } \\
\text { applicable to the online version of the article only. Distribu- } \\
\text { tion permitted for non-commercial purposes only. }\end{array}$ \\
\hline
\end{tabular}

bination enhanced the suppression effects on SNL-induced spinal dorsal horn Fos and OX42 expression, compared to either drug administered alone. These results indicated that combination of tramadol with minocycline could exert synergistic effects on peripheral nerve injury-induced neuropathic pain; thus, a new strategy for treating neuropathic pain by breaking the interaction between neurons and glia bilaterally was also proposed. Copyright $\Theta 2012$ S. Karger AG, Basel

\section{Introduction}

Peripheral nerve injury-induced neuropathic pain is an intractable problem for both clinical treatment and laboratory research. Multiple mechanisms are involved in the initiation and maintenance of nerve injury-induced neuropathic pain, such as hyperexcited primary afferent, abnormal plasticity in spinal dorsal horn, and aberrant neuronal-glial interactions $[1,2]$.

Inhibiting spinal nociceptive neuronal activation after nerve injury is a traditional option for treating neuro-

\section{X.-P.M., L.C., and W.W. contributed equally to this work.}

Hui Zhang, Li-Xian Xu, Yun-Qing Li, Department of Anesthesiology, School of Stomatology and Department of Anatomy, Histology and Embryology, K.K. Leung Brain Research Centre, Fourth Military, Medical University, Xi'an 710032 (PR China) Tel. +86298477 6115, Fax +862984776115

E-Mail fuming@fmmu.edu.cn; kqmzk@126.com; deptanat@fmmu.edu.cn 
pathic pain. Abundant evidence indicated that, as a neuronal activation inhibitor, tramadol was effective in treating neuropathic pain both in the clinic and laboratory $[3$, 4]. Additionally, the clinical concept of a balanced or associative manner proposes the use of a combination of analgesics to provide better pain control [5]. Therefore, studies reported that combined tramadol with some other analgesics exerted better effects on pain relief $[3,6]$. However, all of these combinations just concerned the neuronal participation, but neglected another key factor for neuropathic pain development, glial cells.

Evidence suggested that spinal glial activation was required and sufficient for neuropathic pain processing [79]. By releasing neurotransmitters or some extracellular signaling molecules, and reuptaking neurotransmitters among synaptic cleft, glial cells contribute to the neuronal excitability, synaptic transmission and, thus, coordinating activity in neuronal networks $[1,10]$. Previous studies indicated spinal glial cells (especially microglia and astrocytes) as key factors in the initiation and maintenance of neuropathic pain $[7,8]$. Therefore, suppressing spinal glial activation can be another option for treating neuropathic pain. Minocycline, a second-generation tetracycline, has been used for relieving nerve injury or peripheral inflammation-induced neuropathic pain [11-13]. These effects depend on inhibition of spinal microglial activation and proliferation, which consequently lower the expression of proinflammatory cytokines and neuronal activity.

Many studies indicated that the spinal neuronal and glial interaction was the most important mechanism underlying the development of neuropathic pain and spinal dorsal plasticity $[1,2,14]$. A study showed that spinal ERK was activated in neurons, microglia, and astrocytes sequentially after spinal nerve ligation (SNL), proposing an effect of neuronal activity on glial activation [15]. Glial activation was abolished by blocking primary afferent input with a local anesthetic [2]. Moreover, activated glial cells release a variety of substances, including inflammatory cytokines, chemokines, prostaglandins, brain-derived neurotrophic factor (BDNF), ATP, NO, D-serine, and glutamate, which in turn modulate neuronal activity and facilitate pain transmission [16]. Therefore, it would be better to treat neuropathic pain by a combination of a neuronal inhibitor with a glial modulator or inhibitor to break the 'cross-talk' between neuron and glia [10].

Taken together, the present study hypothesized that combining tramadol with minocycline may exert some additive or synergistic effects on peripheral nerve injuryinduced neuropathic pain and propose a new strategy for clinical therapy.

Tramadol and Minocycline for Neuropathic Pain
To verify this hypothesis, SNL operation was performed to build the neuropathic pain model. Tramadol or minocycline was administered intrathecally to confirm the individual dose-dependent analgesia effect. Based on the dose-effect curve, the ED50 of each drug was calculated. Then, tramadol and minocycline were combined at a fixed ratio according to the ED50. The antiallodynic effect of the drug combination was evaluated with a pain behavioral test. The experimental ED50 and the theoretical ED50 of the drug combination were determined and analyzed with isobolographic analysis to confirm the potential synergistic effects of the drug combination on SNL-induced neuropathic pain. Additionally, immunofluorescence was performed to confirm the effects of these two reagents, administered individually or together, on SNL-induced spinal dorsal horn Fos and OX42 expression.

\section{Materials and Methods}

\section{Animals}

Sprague-Dawley rats (male, $180-200 \mathrm{~g}$ ) were housed in plastic cages and maintained on a 12/12-hour light/dark cycle under conditions of $22-25^{\circ} \mathrm{C}$ ambient temperature with food and water available. All experimental procedures received prior approval (No. 11012) from the Animal Use and Care Committee for Research and Education of the Fourth Military Medical University, Xi'an, China, and the ethical guidelines to investigate experimental pain in conscious animals [17]. All efforts were made to minimize animal suffering and to reduce the number of animals used.

\section{Intrathecal Implantation}

Intrathecal implantation was carried out by inserting polyethylene tubing for injecting drugs directly into the subarachnoid space of the lumbar enlargement. Briefly, a midline incision $(3 \mathrm{~cm})$ was made at the back of the rat from the level of the third thoracic vertebrae to the lower back, under pentobarbital anesthesia ( $45 \mathrm{mg} / \mathrm{kg}$, i.p.). A pre-measured length of polyethylene- $10 \mathrm{tub}$ ing (inside diameter $0.28 \mathrm{~mm}$ and outside diameter $0.61 \mathrm{~mm}$ ) was passed caudally from the T8 to the L 3 level of the spinal cord, and $2 \mathrm{~cm}$ of the free ending was left exposed in the upper thoracic region. Rats were allowed to recover for 3-5 days before further use. Only the animals judged as neurologically normal and that showed complete paralysis of the tail and bilateral hind legs after administration of $2 \%$ lidocaine $(10 \mu \mathrm{l})$ through the intrathecal catheter were used for the following experiments.

\section{Spinal Nerve Ligation}

With pentobarbital anesthesia ( $45 \mathrm{mg} / \mathrm{kg}$, i.p.), the L6 vertebra left transverse process of the animal was first removed to expose the L4 and L5 spinal nerves. The L5 spinal nerve was then carefully isolated and tightly ligated with 6-0 silk thread [18]. The surgical procedure for the sham group was identical to that of the SNL group, except that the spinal nerve was not ligated. 
Nociceptive Behavioral Tests

Animals were habituated to the testing environment for 3 days before the baseline test and were then placed under inverted plastic boxes $(30 \times 30 \times 50 \mathrm{~cm})$ on an elevated mesh floor for $30 \mathrm{~min}$ before the threshold testing to allow habituation. Briefly, a logarithmic series of 8 calibrated Semmes-Weinstein monofilaments (von-Frey hairs; Stoelting, Kiel, Wisc., USA) were applied to the ipsilateral hind paw to determine the stimulus intensity threshold stiffness required to elicit a paw withdrawal response. Log stiffness of the hairs is determined by $\log 10(\mathrm{mg} \times 10)$ [19]. The 8 filaments had the following log stiffness values (value in grams is given in parentheses): 4.17 (1,479 mg), $4.31(2,041 \mathrm{mg}), 4.56(3,630$ $\mathrm{mg}), 4.74$ (5,495 mg), 4.93 (8,511 mg), 5.07 (11,749 mg), 5.18 (15,136 $\mathrm{mg})$, and $5.46(28,840 \mathrm{mg})$. The range of monofilaments $(1.479$ $28.840 \mathrm{mg}$ ) produced a logarithmically graded slope when interpolating a $50 \%$ response threshold of stimulus intensity (expressed as $\left.\log _{10}(\mathrm{mg} \times 10)\right)$ [20]. The behavioral responses were used to calculate the $50 \%$ paw withdrawal threshold, by fitting a Gaussian integral psychometric function using a maximum-likelihood fitting method, as described in detail previously [19]. This fitting method allowed parametric statistical analysis. The percentage of the antiallodynia was calculated according to the following equation [21]: \% antiallodynia $=100-100 \times$ (baseline of SNL-drug - post-SNL-drug)/(baseline of SNL-saline - post-SNLsaline). All behavioral tests were performed in a double-blind manner.

\section{Intrathecal Drug Administration}

Tramadol hydrochloride and minocycline hydrochloride (Sigma) were dissolved and diluted with preservative-free normal saline solution for administration. Normal saline $(0.9 \%)$ was used as the negative control. Tramadol was injected in doses of 3,10 , and $30 \mu \mathrm{g} / \mathrm{rat}$, and minocycline in doses of 10,30 , and $100 \mu \mathrm{g} / \mathrm{rat}$. The dose-effect curve was constructed and the experimental points fitted using least-square linear regression. Then, the ED50 (50\% antiallodynia) of each drug was calculated [22]. To assess interaction between drugs, tramadol and minocycline were administered in fixed ratio combination (tramadol ED50/2 + minocycline ED50/2 $\mu \mathrm{g} / \mathrm{rat}$; tramadol ED50/4 + minocycline ED50/4 $\mu \mathrm{g} / \mathrm{rat}$, and tramadol ED50/8 + minocycline ED50/8 $\mu \mathrm{g} / \mathrm{rat}$ ). Drugs and saline $(10 \mu \mathrm{l}$ each) were injected intrathecally over $30 \mathrm{~s}$, followed by a $10-\mu \mathrm{l}$ flush of normal saline.

\section{Rotarod Test}

In order to assess whether the drugs used in the present experiment could influence motor function, which might influence the behavioral results, we performed rotarod tests on animals with intrathecal drug administration but without SNL operation and von Frey test. Rats with no previous exposures to the rotarod test were placed on the Ugo Basile 7650 Rotarod accelerator treadmill (Ugo Basile, Varese, Italy) set at the minimal speed for training sessions of 1-2 min at intervals of 30-60 min. After this learning period, the animals were placed onto the rotarod at a constant speed of 25 r.p.m. As the animal took a grip of the drum, the accelerator mode was selected on the treadmill, i.e. the rotation rate of the drum was increased linearly at 20 r.p.m. Thereafter, the time was measured from the start of the acceleration period until the rat fell off the drum. The cutoff time was $30 \mathrm{~s}$. Each rat was tested $30 \mathrm{~min}$ before drug administration as control performance and then once a day for 4 days during the drug administration period. The time that the animal remained on the rotarod was recorded and expressed as a percentage of that animal's own mean control performance.

\section{Immunofluorescence}

After deep anesthesia with pentobarbital ( $60 \mathrm{mg} / \mathrm{kg}$, i.p.), the rats were perfused through the ascending aorta with $100 \mathrm{ml}$ $0.9 \%$ saline followed by $500 \mathrm{ml} 0.1 \mathrm{M}$ phosphate buffer $(\mathrm{PB}, \mathrm{pH}$ 7.3) that contained $4 \%$ paraformaldehyde and $2 \%$ picric acid. After perfusion, the L5 spinal segment was removed and postfixed in the same fixative for $2-4 \mathrm{~h}$ and then cryoprotected for $24 \mathrm{~h}$ at $4^{\circ} \mathrm{C}$ in $0.1 \mathrm{M} \mathrm{PB}$ that contained $30 \%$ sucrose. Transversefrozen spinal sections ( $30 \mu \mathrm{m}$ thick) were cut with a cryostat (Leica CM1800; Heidelberg, Germany) and collected serially in three dishes. Each dish contained a complete set of serial sections that were processed for immunofluorescent staining. One of the dishes was selected randomly. The sections in the dish were rinsed in $0.01 \mathrm{M}$ phosphate-buffered saline (PBS, $\mathrm{pH}$ 7.3) 3 times (10 min each), blocked with $2 \%$ goat serum in $0.01 \mathrm{M} \mathrm{PBS}$ that contained $0.3 \%$ Triton $\mathrm{X}-100$ for $1 \mathrm{~h}$ at room temperature (RT, $20-25^{\circ} \mathrm{C}$ ), and then used for immunofluorescent staining. The sections were incubated overnight at $4^{\circ} \mathrm{C}$ with primary antibody: mouse anti-OX42 (1:500; Abcam, Cambridge, UK) or mouse anti-Fos (1:500; Abcam). The sections were washed 3 times in $0.01 \mathrm{M}$ PBS (10 min each) and then incubated for $4 \mathrm{~h}$ at RT with the secondary antibody: Alex 488-conjugated horse anti-mouse IgG (1:500; Vector, Burlingame, Calif., USA) or Alex 594 donkey anti-mouse IgG (1:500; Invitrogen, Carlsbad, Calif., USA). The specificity of the staining was tested on the sections in another dish by omission of the primary specific antibodies. No immunoreactive products were found on the sections (data not shown). Confocal images were obtained using a confocal laser microscope (FV1000; Olympus, Tokyo, Japan) and digital images were captured with Fluoview 1000 (Olympus). For semiquantification, the fluorescent brightness value of OX42 or Foslike immunoreactivity was detected on the same areas of the dorsal horn by using software under IX-70 confocal microscope. After the images were captured, optical density of the same areas of the ipsilateral superficial dorsal horn (laminae I and II) was calculated [23]. The relative value of OX42 or Fos immunodensity was expressed as percentage changes compared to that of sham-saline control.

\section{Quantification and Statistical Analysis}

All data were collected by researchers blinded to the surgery and reagents used. Data from the von Frey test were presented as means \pm SD and analyzed as the interpolated 50\% threshold (absolute threshold) in log base 10 of stimulus intensity (monofilament stiffness in $\mathrm{mg} \times 10$ ). Repeated measures ANOVA (with Bonferroni confidence interval adjustment) was used and conducted for analyzing. Data from the rotarod test were presented as means \pm SD. Repeated measures ANOVA (with Bonferroni confidence interval adjustment) was used and conducted for analyzing. Data from immunofluorescence were expressed as means $\pm \mathrm{SD}$. Differences in changes of values over time of each group were tested using one-way ANOVA, followed by the least significant difference test.

Isobolographic analysis was used for evaluating the interaction after drug coadministration $[21,22,24]$. In brief, ED50 of each drug was calculated by linear regression. A combination of 


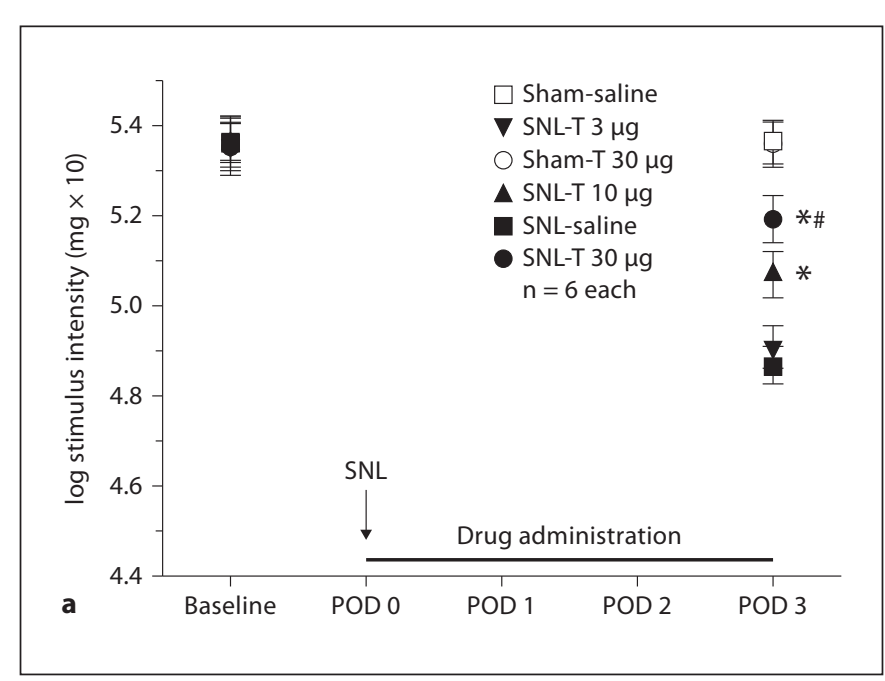

Fig. 1. Antiallodynic effect of intrathecal tramadol on SNL-induced neuropathic mechanical allodynia. a Dose-dependent effect of intrathecal tramadol on SNL-induced mechanical allodynia. Intrathecal tramadol (10 or $30 \mu \mathrm{g} / \mathrm{rat}$ ) obviously elevated the pain threshold, whereas intrathecal tramadol $3 \mu \mathrm{g} / \mathrm{rat}$ did not show an apparent effect. $\mathbf{b}$ Percentage of antiallodynia of the max-

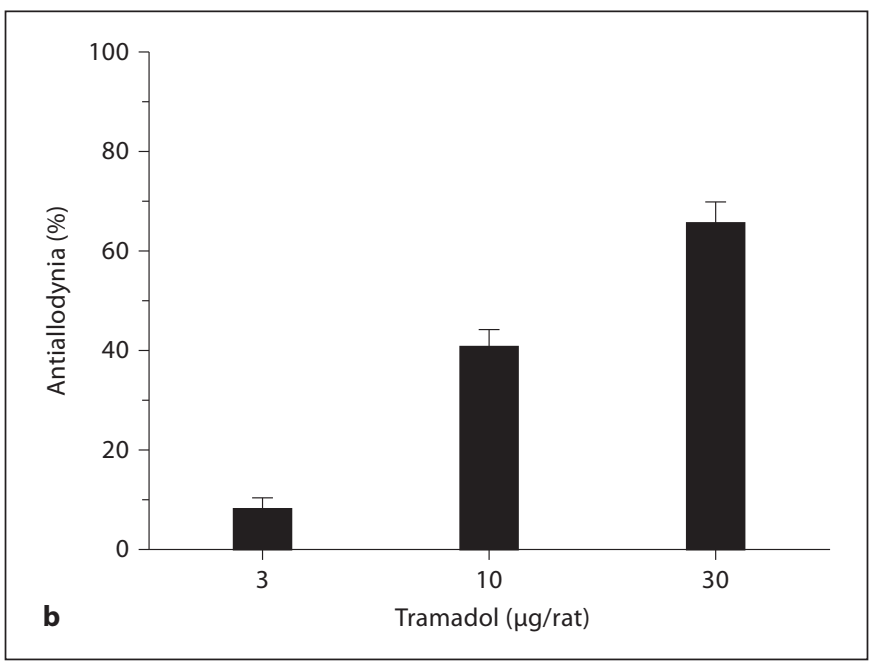

imum possible effect. The y-axis is the percentage of antiallodynia after drug administration: \% antiallodynia $=100-100 \times$ (baseline of SNL-drug - post-SNL-drug)/(baseline of SNL-saline - post-SNL-saline). ${ }^{*} \mathrm{p}<0.05$, compared with that of SNL-saline. \# $\mathrm{p}<0.05$, compared with that of SNL-T $10 \mu \mathrm{g}$ group. Six rats in each group. $\mathrm{T}=$ Tramadol. the two drugs was administered in a constant dose ratio based on ED50 values (tramadol ED50/2 + minocycline ED50/2 $\mu \mathrm{g} /$ rat; tramadol ED50/4 + minocycline ED50/4 $\mu \mathrm{g} / \mathrm{rat}$, and tramadol ED50/8 + minocycline ED50/8 $\mu \mathrm{g} / \mathrm{rat})$. For drug combination, the theoretic ED50 is tramadol ED50/2 + minocycline ED50/2. Experimental values of drug combination from fixed ratio-designed studies were also analyzed using linear regression, and then the experimental ED50 value of drug combination was calculated $(50 \%$ antiallodynia in SNL-induced mechanical allodynia). The statistical significance between the theoretical ED50 and experimental ED50 of drug combination was evaluated with Student's t test. An experimental ED50 significantly less than the theoretical ED50 was considered to indicate a synergistic interaction between tramadol and minocycline.

All statistical analyses were performed using SPSS ${ }^{\circledR}$ version 16.0 software (SPSS Inc., Chicago, Ill., USA). $\mathrm{p}<0.05$ was considered statistically significant.

\section{Experimental Procedures}

All rats received intrathecal intubation and were allowed to recover for 3-5 days before further use. The baseline value of the behavioral test was carried out on the SNL or sham surgical day prior to the beginning of drug administration. The SNL or sham surgical procedure was carried out after drug administration on post-operative day (POD) 0 . Drugs were applied for 4 days from POD 0 to 3 . Then, the behavioral tests were performed on POD 3 after drug administration. Finally, all rats were sacrificed for immunofluorescent study after the behavioral test on POD 3.

Tramadol and Minocycline for Neuropathic Pain

\section{Results}

Intrathecal Tramadol or Minocycline Attenuated

SNL-Induced Mechanical Allodynia in a

Dose-Dependent Manner Individually

In order to detect the effects of intrathecal tramadol or minocycline on SNL-induced neuropathic pain, these two reagents were injected individually with three different concentrations for 4 days from the SNL operation day to POD 3. The changes of the mechanical pain threshold were observed on POD 3.

The baseline value of each group did not show any statistical difference.

Intrathecal application of $3 \mu \mathrm{g}$ tramadol from POD 0 to 3 had no obvious effect on SNL-induced mechanical allodynia (fig. 1a). Intrathecal tramadol $10 \mu \mathrm{g}$ elevated the pain threshold significantly, compared to that of the SNL-saline group (fig. 1a, $\mathrm{p}<0.05$ ). A higher dose of tramadol $(30 \mu \mathrm{g})$ relieved SNL-induced mechanical allodynia apparently on POD 3 (fig. 1a, p $<0.05$, compared to that of the SNL-saline control). Besides, a 30- $\mu$ g tramadol application showed a stronger effect on antiallodynia than a $10-\mu$ g tramadol administration (fig. 1a, p < 0.05). However, the pain threshold of sham rats was not affect by intrathecal tramadol even with $30 \mu \mathrm{g}$ administration. The dose-effect response suggested that intrathe- 


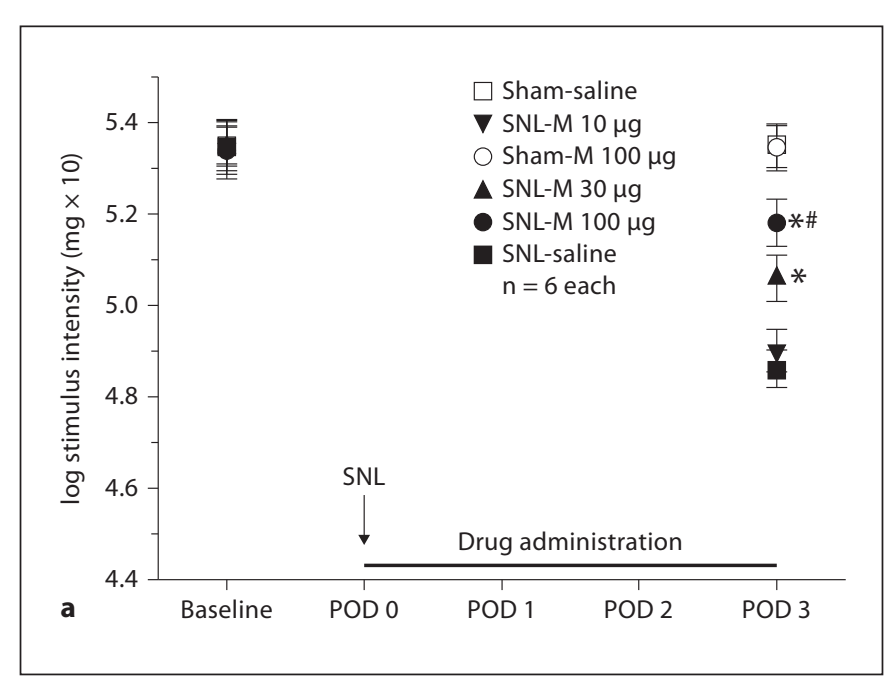

Fig. 2. Antiallodynic effect of intrathecal minocycline on SNLinduced neuropathic mechanical allodynia. a Dose-dependent effect of intrathecal minocycline on SNL-induced neuropathic pain. Intrathecal minocycline (30 or $100 \mu \mathrm{g} / \mathrm{rat}$ ) apparently raised the pain threshold, whereas intrathecal minocycline $10 \mu \mathrm{g} / \mathrm{rat}$ did not show an obvious effect on SNL-induced allodynia. b Percent-

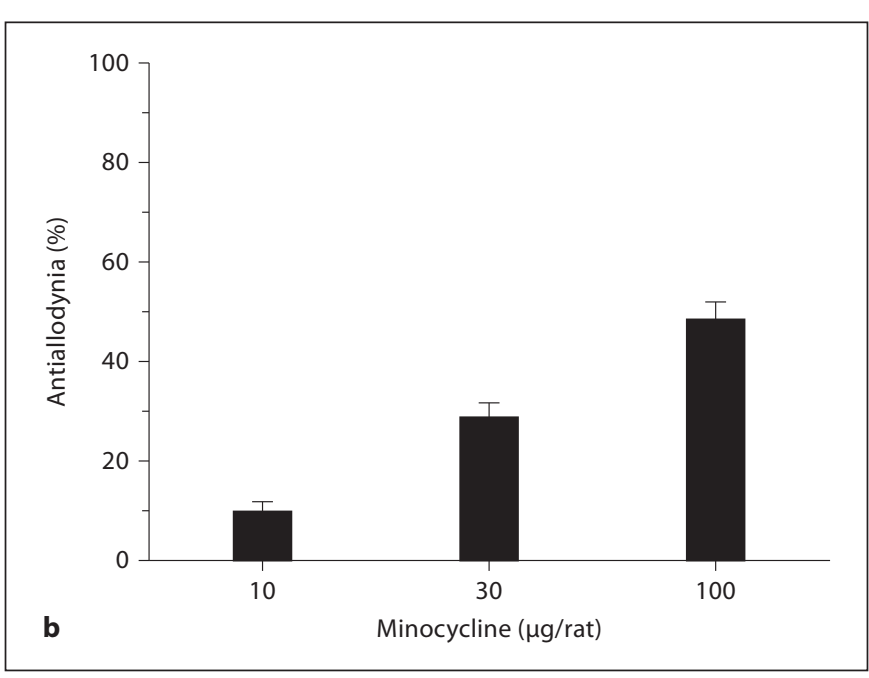

age of antiallodynia of the maximum possible effect. The y-axis is the percentage of antiallodynia after drug administration: \% antiallodynia $=100-100 \times$ (baseline of SNL-drug - post-SNLdrug)/(baseline of SNL-saline - post-SNL-saline). ${ }^{*} \mathrm{p}<0.05$, compared with that of SNL-saline. ${ }^{\#} p<0.05$, compared with that of SNL-M $30 \mu$ group. Six rats in each group. $M=$ Minocycline.

Table 1. Dose used in the study of the interaction between tramadol and minocycline after intrathecal application

\begin{tabular}{|c|c|c|c|c|c|}
\hline \multicolumn{2}{|c|}{ Tramadol in combination } & \multicolumn{2}{|c|}{ Minocycline in combination } & \multicolumn{2}{|c|}{ Total dose } \\
\hline dose, $\mu \mathrm{g}$ & antiallodynia, \% & dose, $\mu \mathrm{g}$ & antiallodynia, $\%$ & dose, $\mu \mathrm{g}$ & $\begin{array}{l}\text { antiallodynia, \% } \\
\text { (theoretically) }\end{array}$ \\
\hline 15.5 & $50.0(\mathrm{ED} 50)$ & 109.2 & $50.0(\mathrm{ED} 50)$ & & \\
\hline 7.8 & $25.0(\mathrm{ED} 50 / 2)$ & 54.6 & $25.0(\mathrm{ED} 50 / 2)$ & 62.4 & $50.0(\mathrm{ED} 50)$ \\
\hline 3.9 & $12.5(\mathrm{ED} 50 / 4)$ & 27.3 & $12.5(\mathrm{ED} 50 / 4)$ & 31.2 & $25.0(\mathrm{ED} 50 / 2)$ \\
\hline 1.9 & $6.25(\mathrm{ED} 50 / 8)$ & 13.7 & $6.25(\mathrm{ED} 50 / 8)$ & 15.6 & $12.5(\mathrm{ED} 50 / 4)$ \\
\hline
\end{tabular}

cal tramadol $(3,10$, or $30 \mu \mathrm{g})$ demonstrated an effective and reliable antiallodynia effect in a dose-dependent manner on SNL-induced neuropathic mechanical allodynia (fig. 1b). Additionally, the ED50 dose (50\% antiallodynia) of intrathecal tramadol was evaluated according to the dose-effect response by liner regression analysis (table 1).

Intrathecal minocycline $10 \mu \mathrm{g}$ did not produce any effects on SNL-induced neuropathic pain. Minocycline 30 $\mu \mathrm{g}$ could attenuate SNL-induced neuropathic pain after administration on POD 3 (fig. 2a, p $<0.05$, compared to that of the SNL-saline group). Moreover, a higher dose of minocycline $(100 \mu \mathrm{g})$ significantly suppressed SNL-in- duced mechanical allodynia, compared to that of the SNL-saline group (fig. $2 \mathrm{a}, \mathrm{p}<0.05$ ). Furthermore, the antiallodynia effect of $100 \mu \mathrm{g}$ minocycline was more effective than that of $30 \mu \mathrm{g}$ minocycline application to nerve injury animals (fig. $2 \mathrm{a}, \mathrm{p}<0.05$ ). However, even $100 \mu \mathrm{g}$ of minocycline application could not change the pain threshold of the sham operation rats. The dose-effect response indicated that intrathecal minocycline $(10,30$, or $100 \mu \mathrm{g})$ exerted an antiallodynia effect in a dose-dependent manner on SNL-induced neuropathic pain (fig. 2b). Finally, the ED50 dose (50\% antiallodynia) of intrathecal minocycline was calculated in accordance with the doseeffect response by liner regression analysis (table 1). 


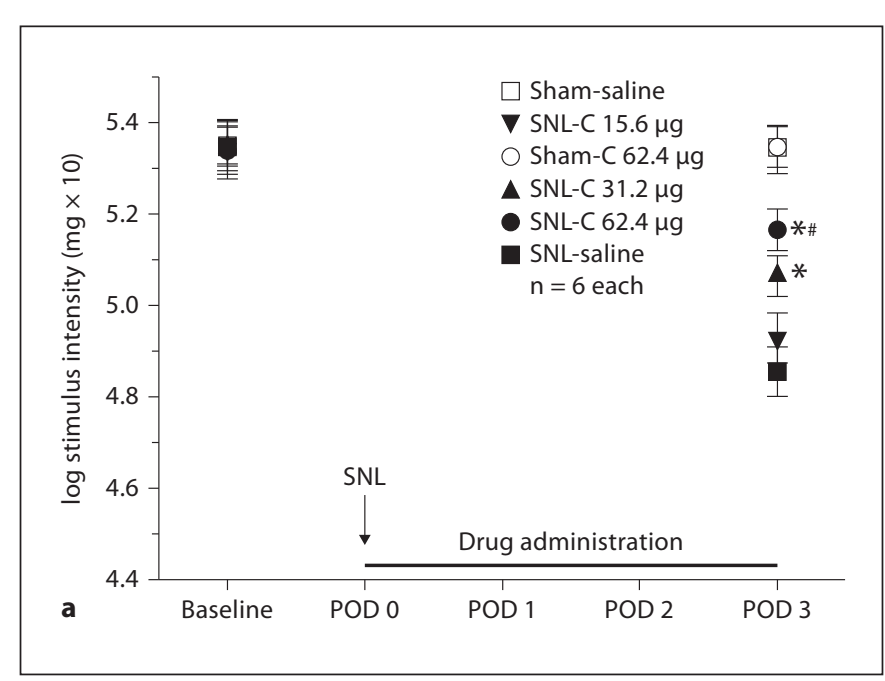

Fig. 3. Antiallodynic effect of intrathecal tramadol and minocycline combination on SNL-induced neuropathic pain. a Dose-dependent effect of drug combination on SNL-induced mechanical allodynia. Coadministration of $62.4 \mu \mathrm{g} / \mathrm{rat}(7.8 \mu \mathrm{g} / \mathrm{rat}$ tramadol and $54.6 \mu \mathrm{g} / \mathrm{rat}$ minocycline) remarkably reversed the mechanical allodynia. Combination of $31.2 \mu \mathrm{g} / \mathrm{rat}(3.9 \mu \mathrm{g} / \mathrm{rat}$ tramadol and $27.3 \mu \mathrm{g} /$ rat minocycline) also effectively elevated the pain threshold. However, intrathecal $15.6 \mu \mathrm{g} / \mathrm{rat}(1.9 \mu \mathrm{g} / \mathrm{rat}$ tramadol

\section{Effects of Tramadol and Minocycline Combination on}

SNL-Induced Neuropathic Mechanical Allodynia

In order to investigate the effects of drug combination on SNL-induced mechanical allodynia, tramadol and minocycline were co-applied in a constant dose ratio based on ED50 values (tramadol ED50/2 + minocycline ED50/2, tramadol ED50/4 + minocycline ED50/4, and tramadol ED50/8 + minocycline ED50/8 $\mu \mathrm{g} / \mathrm{rat})$. Therefore, the doses in combination were 62.4, 31.2, and $15.6 \mu \mathrm{g}$ (table 1).

The baseline value of each group did not show any statistical difference. Coadministration of $15.6 \mu \mathrm{g}$ of the drugs did not show any obvious effects on SNL-induced neuropathic pain. Drug combination of $31.2 \mu \mathrm{g}$ significantly elevated the pain threshold of the nerve injury animals compared with that of the SNL-saline group (fig. 3a, $\mathrm{p}<0.05)$. Furthermore, injection of $62.4 \mu \mathrm{g}$ of the drugs in combination demonstrated a markedly powerful effect on SNL-induced mechanical allodynia, compared to that of the SNL-saline group (fig. 3a, p < 0.05). However, application of $62.4 \mu \mathrm{g}$ of the drugs in combination had no effect on sham-operated rats. The dose-effect response of drug combination demonstrated that tramadol and minocycline combination generated an antiallodynic effect on SNL-induced neuropathic pain also in a dose-dependent manner (fig. 3b). According to the dose-effect response, the

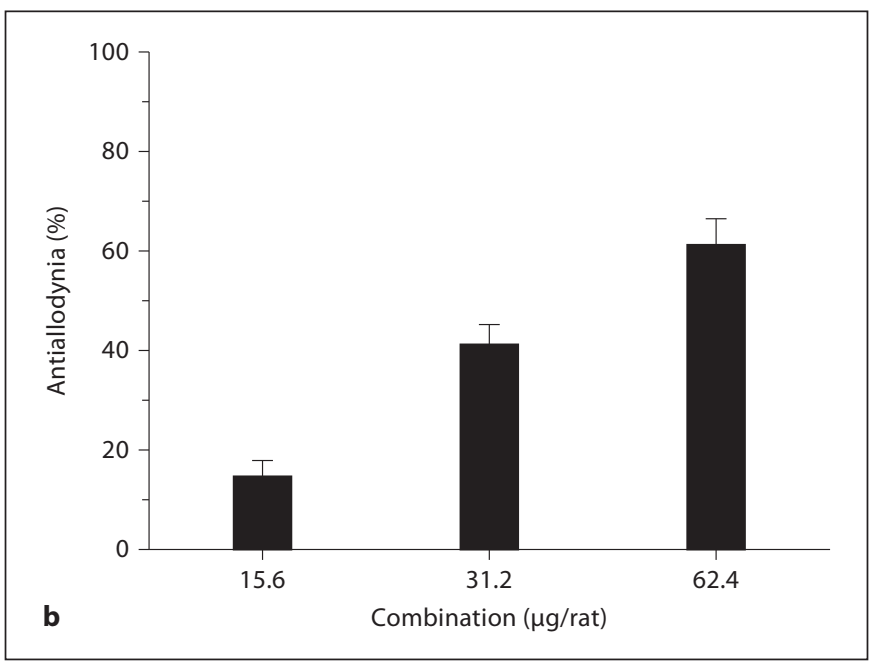

and $13.7 \mu \mathrm{g} /$ rat minocycline) had no obvious effect on mechanical allodynia. $\mathbf{b}$ Percentage of antiallodynia of the maximum possible effect. The $y$-axis is the percentage of antiallodynia after drug administration: $\%$ antiallodynia $=100-100 \times$ (baseline of SNL-drug - post-SNL-drug)/(baseline of SNL-saline - post-SNLsaline). ${ }^{*} \mathrm{p}<0.05$, compared with that of SNL-saline. ${ }^{*} \mathrm{p}<0.05$, compared with that of SNL-C $31.2 \mu \mathrm{g}$ group. Six rats in each group. $\mathrm{C}=$ Combination.

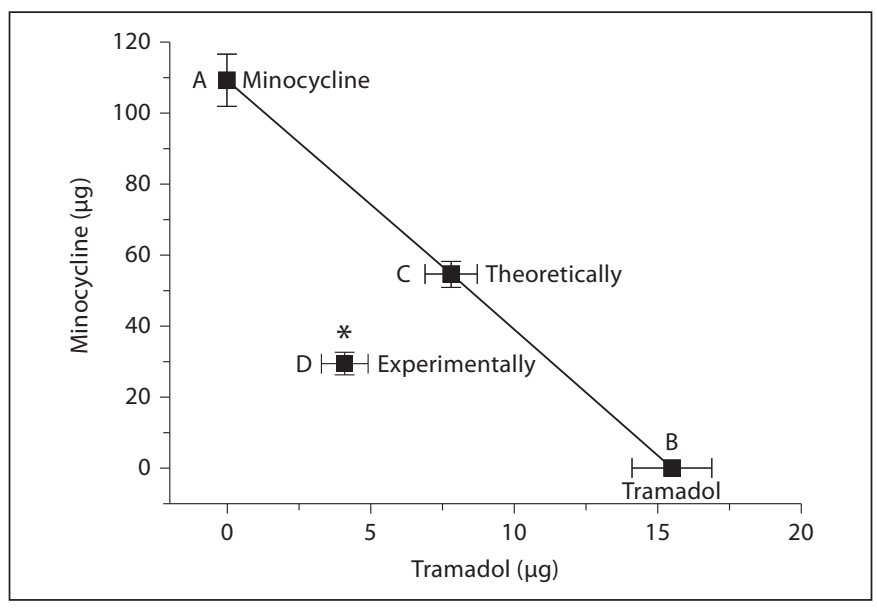

Fig. 4. Isobologram of drug combination shows the synergistic effect of intrathecal tramadol and minocycline coadministration on SNL-induced neuropathic mechanical allodynia. A and B indicate the ED50 of intrathecal minocycline or tramadol, respectively. The oblique line between A and B is the theoretic additive effect line of tramadol and minocycline coadministration. $\mathrm{C}$, in the middle of the line, is the theoretical ED50 of the drug combination, which is calculated from the individual drug ED50. D, far below the line, is the experimental ED50 of the drug combination, which is actually observed after drug coadministration. The experimental ED50 point lies far below the additive line, suggesting a significant synergistic effect of drug coadministration. ${ }^{*} \mathrm{p}<$ 0.05 , compared with that of theoretical ED50. 
experimental ED50 dose (50\% antiallodynia) of drug combination could be calculated by liner regression analysis.

To confirm whether tramadol and minocycline combination could exert some synergistic effects on SNL-induced neuropathic pain, the isobolographic analysis was carried out for comparing the theoretical ED50 with experimental ED50 of drugs combination (fig. 4). The theoretical additive line indicates that all points of tramadol and minocycline combinations in this line produce an effect of theoretical 50\% antiallodynia (theoretical ED50) according to an additive interaction (fig. 4). The experimental 50\% antiallodynia (experimental ED50) value of tramadol and minocycline combination was located below the theoretical additive line, suggesting synergistic effects of tramadol and minocycline combination on SNL-induced neuropathic mechanical allodynia. The significant difference between experimental ED50 and theoretical ED50 was verified by the Student's t test (fig. $4, \mathrm{p}<0.05$ ).

\section{Effects of Drug Combination on Suppression of SNL-Induced Spinal Dorsal Horn Fos and \\ OX42 Expression}

SNL upregulated Fos expression remarkably in the spinal dorsal horn compared to that of the sham-saline group (fig. 5a, b). Intrathecal tramadol $(30 \mu \mathrm{g})$ alone significantly downregulated Fos expression compared to that of the SNL-saline group (fig. 5c). Moreover, both tramadol $10 \mu \mathrm{g}$ and minocycline $30 \mu \mathrm{g}$ could suppress SNL-induced Fos expression compared to that of the SNL-saline group (fig. 5d, e). Additionally, drug combination (tramadol $3.9 \mu \mathrm{g}$ and minocycline $27.3 \mu \mathrm{g}$ ) generated a more powerful inhibiting effect on SNL-induced Fos expression than tramadol $10 \mu \mathrm{g}$ or minocycline $30 \mu \mathrm{g}$ given alone (fig. 5f). The statistical differences between groups were analyzed with one-way ANOVA (fig. 5g, p < 0.05).

SNL induced notable microglial activation, shown by OX42 upregulation in the ipsilateral spinal dorsal horn of the SNL-saline group (fig. 6a). Intrathecal minocycline $(100$ and $30 \mu \mathrm{g})$ inhibited SNL-induced microglial activation in a dose-dependent manner (fig. 6c, d). Moreover, intrathecal tramadol $10 \mu \mathrm{g}$ could also downregulate microglial activation (fig. 6e). SNL-induced spinal dorsal horn microglial activation was apparently suppressed by drug coadministration (tramadol $3.9 \mu \mathrm{g}$ and minocycline $27.3 \mu \mathrm{g}$ ) compared to tramadol $10 \mu \mathrm{g}$ or minocycline $30 \mu \mathrm{g}$ given alone (fig. $6 \mathrm{f}$ ). The statistical differences between groups were analyzed with one-way ANOVA (fig. 6g, p < 0.05).

These results indicated that tramadol and minocycline combination could facilitate the suppressive effect of tramadol on SNL-induced spinal dorsal neuronal activity and enhance the inhibiting effect of minocycline on SNL-induced spinal microglial activation.

\section{Effects of Drugs on Motor Functions Indicated by} the Rotarod Test

Motor dysfunctions would interfere with nociceptive behavioral results. In order to assess whether the drugs (each in high dose: tramadol $30 \mu \mathrm{g}$, minocycline $100 \mu \mathrm{g}$, or drug combination $62.4 \mu \mathrm{g}$ ) used in the present study could impair motor functions, animals with intrathecal drug administration but without SNL operation and von Frey test were assessed with the rotarod test. Repeated drug administration (tramadol $30 \mu \mathrm{g}$, minocycline 100 $\mu \mathrm{g}$, or drug combination $62.4 \mu \mathrm{g}$ ) did not affect the motor performance of rats compared with their own baseline (fig. 7).

\section{Discussion}

Both tramadol and minocycline were reported to be useful for treating neuropathic pain [25]. However, it is unclear whether tramadol and minocycline could be combined for coadministration and what interactive effect could be generated after combination. The present study showed that intrathecal administration of tramadol or minocycline could relieve SNL-induced mechanical allodynia in a dose-dependent manner individually. Combination of tramadol with minocycline indicated synergistic antiallodynic effects on SNL-induced neuropathic pain also in a dose-dependent manner. Moreover, coadministration of tramadol and minocycline facilitated each other's effects on inhibiting SNL-induced spinal dorsal horn Fos expression and microglial activation. Taken together, the present study suggested that combination of tramadol and minocycline could exert some synergistic effects on neuropathic pain by suppressing neuronal and glial activation bilaterally, which may propose a new strategy for treating peripheral nerve injuryinduced neuropathic pain.

\section{Tramadol Played as a Neuronal Activation Inhibitor} when Treating Neuropathic Pain

Both clinical and basic studies indicated that intrathecal tramadol was useful for pain control [26, 27], especially for treating neuropathic pain $[3,4]$. In accordance with previous reports, the present study showed that intrathecal tramadol attenuated SNL-induced neuropathic pain in a dose-dependent manner. Tramadol is known as 

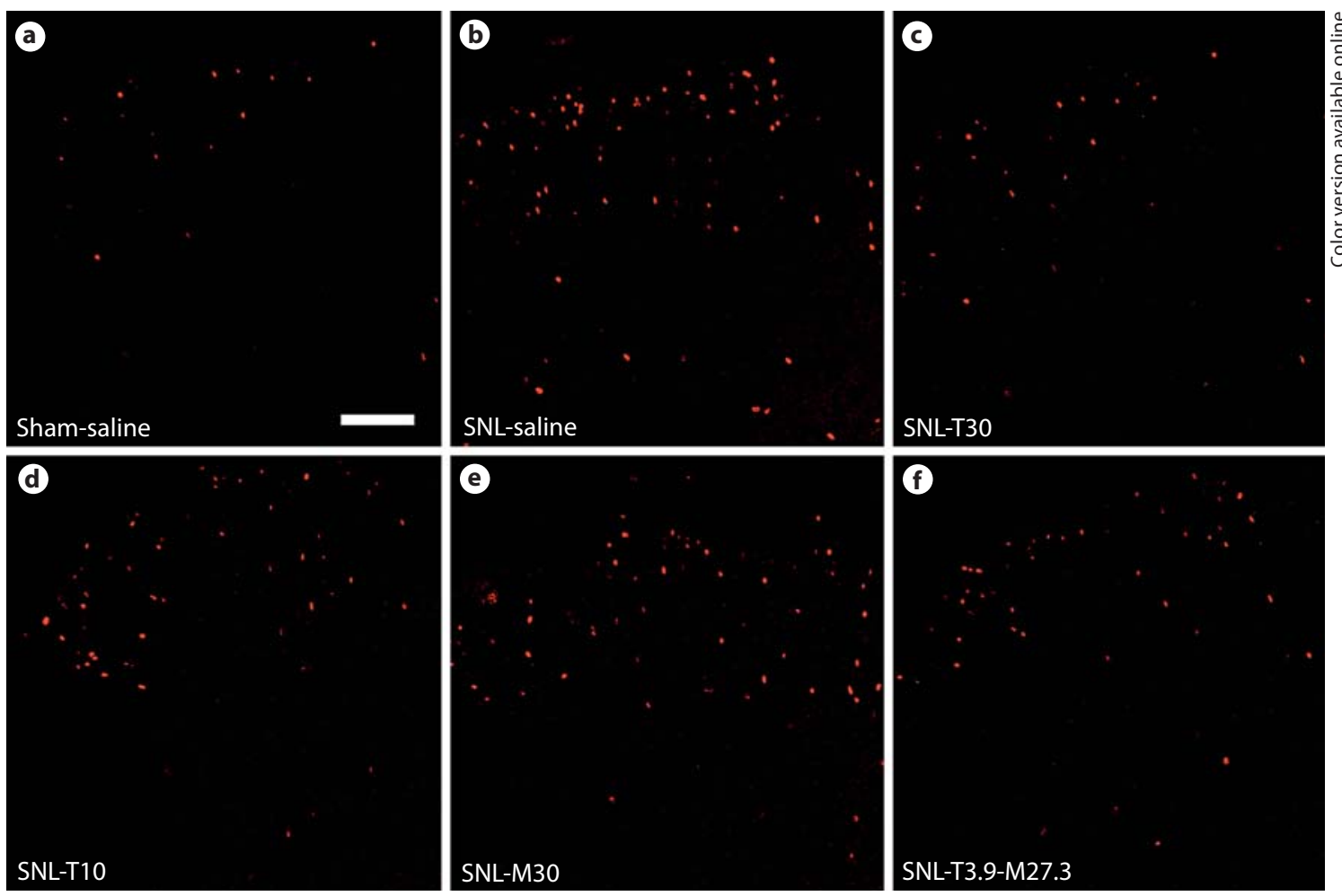

Fig. 5. Effects of drug administration on SNL-induced spinal Fos expression. SNL induced an obvious spinal Fos up-expression in the spinal dorsal horn (b) compared with that of the sham-saline group (a). Intrathecal tramadol (30 and $10 \mu \mathrm{g} / \mathrm{rat}$ ) exhibited suppression effects on SNL-induced Fos expression in a dose-dependent manner $(\mathbf{c}, \mathbf{d})$. Intrathecal minocycline $30 \mu \mathrm{g} / \mathrm{rat}$ also downregulated Fos expression (e). Combination of tramadol $(3.9 \mu \mathrm{g} /$ rat) and minocycline $(27.3 \mu \mathrm{g} / \mathrm{rat})$ showed a remarkable inhibiting effect on SNL-induced spinal Fos expression (f). g Statistical analysis of the spinal Fos expression after different treatments. The y-axis is the relative value of Fos immunodensity, which is expressed as percentage changes compared to that of the shamsaline control group. ${ }^{*}$ Statistically significant difference with $\mathrm{p}<$ 0.05 between groups. Six rats in each group. $\mathrm{T}=$ Tramadol; $\mathrm{M}=$ minocycline. Scale bar $=100 \mu \mathrm{m}$.

an opioid receptor agonist, which could act on the $\mu$-opioid receptor and suppress pain-induced spinal dorsal horn neuronal activation [28]. Studies indicate that peripheral nerve injury-induced neuropathic pain could be relieved by an opioid receptor agonist, such as morphine, tramadol, etc. $[29,30]$. In addition, intrathecal pretreatment with an equi-effective dose of the $\mu$-opioid receptor

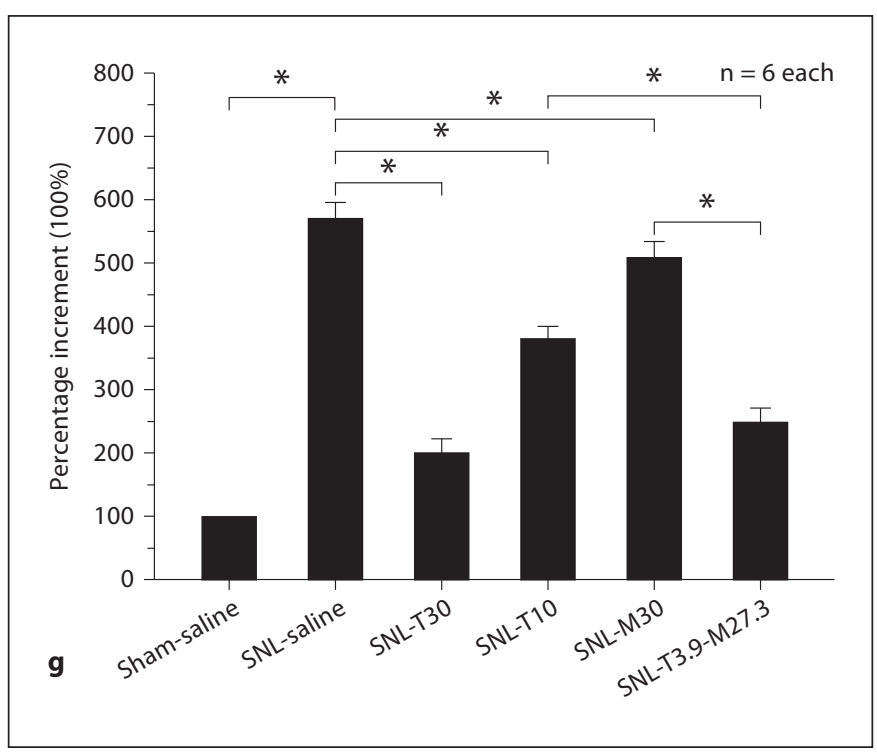

agonist could completely prevent spinal Fos expression, especially in the spinal dorsal horn [31]. Fos is expressed in the nuclei of nociceptive neurons following various noxious stimuli, thus serving as a marker for activated neurons. Increased Fos immunoreactivity directly reflects upregulated neuronal activity [32]. Previous reports indicated that while peripheral nerve injury-in- 

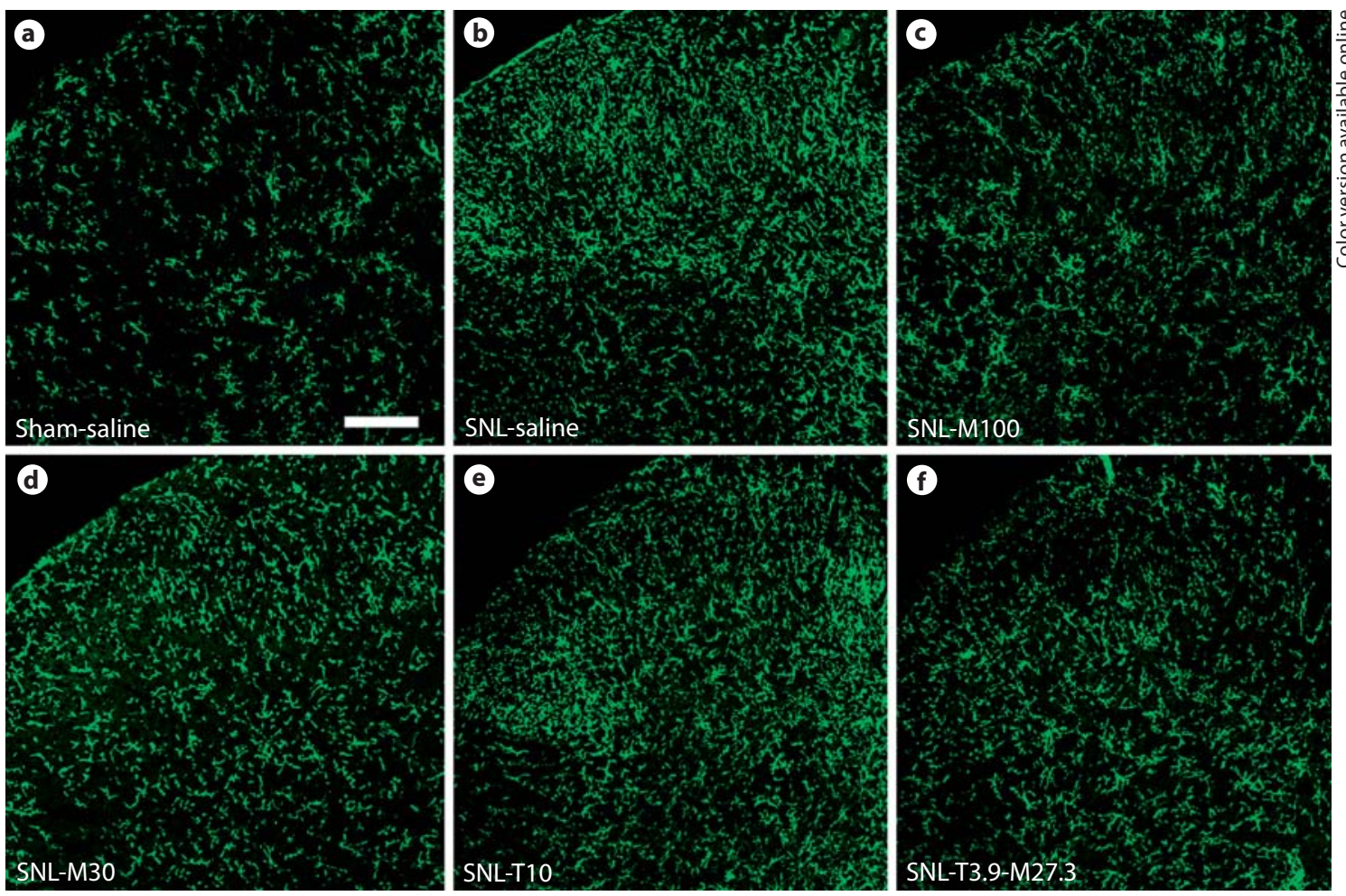

Fig. 6. Effects of drug application on SNL-induced spinal microglial activation. SNL induced a remarkable spinal microglial activation indicated by OX42 up-expression in the spinal dorsal horn (b) compared with that of the sham-saline group (a). Intrathecal minocycline (100 and $30 \mu \mathrm{g} / \mathrm{rat}$ ) inhibited SNL-induced spinal microglial activation in a dose-dependent manner (c, d). Tramadol $10 \mu \mathrm{g} / \mathrm{rat}$ could also cut down the SNL-induced spinal microglial activation level after intrathecal administration (e). Drug coadministration (tramadol $3.9 \mu \mathrm{g} / \mathrm{rat}$ and minocycline $27.3 \mu \mathrm{g} /$ rat) displayed a notable suppressing effect on SNL-induced spinal microglial activation (f). $\mathbf{g}$ Statistical analysis of SNL-induced spinal microglial activation after different treatments. The y-axis is the relative value of OX42 immunodensity, which is expressed as percentage changes compared to that of the sham-saline control group. ${ }^{*}$ Statistically significant difference with $\mathrm{p}<0.05$ between groups. Six rats in each group. $\mathrm{T}=$ Tramadol; $\mathrm{M}=$ minocycline. Scale bar $=100 \mu \mathrm{m}$.

duced neuropathic pain was evoked, Fos-labeled neurons were significantly increased in the spinal dorsal horn. Analgesic therapeutics alleviated neuropathic pain, suppressing the increase of Fos-labeled cells in the spinal dorsal horn $[33,34]$. Besides, our previous study also

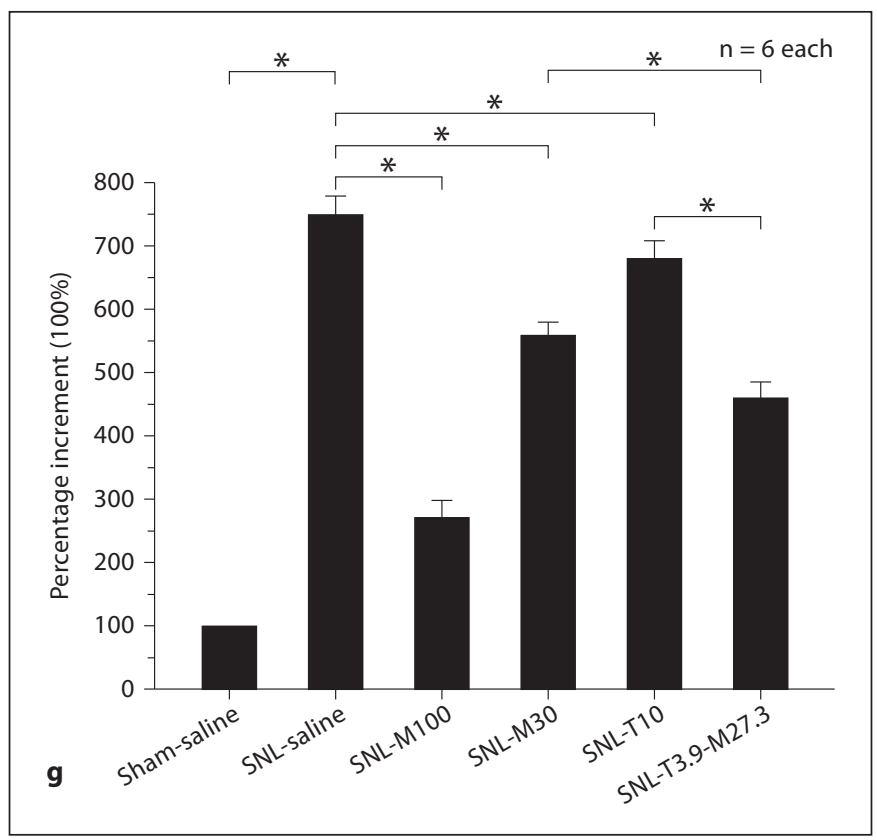

confirmed that spinal Fos expression could be used as a positive marker for activated neurons in the model of SNL-induced neuropathic pain [23]. The present study observed that intrathecal tramadol could suppress SNLinduced spinal dorsal horn Fos expression in a dose-de- 


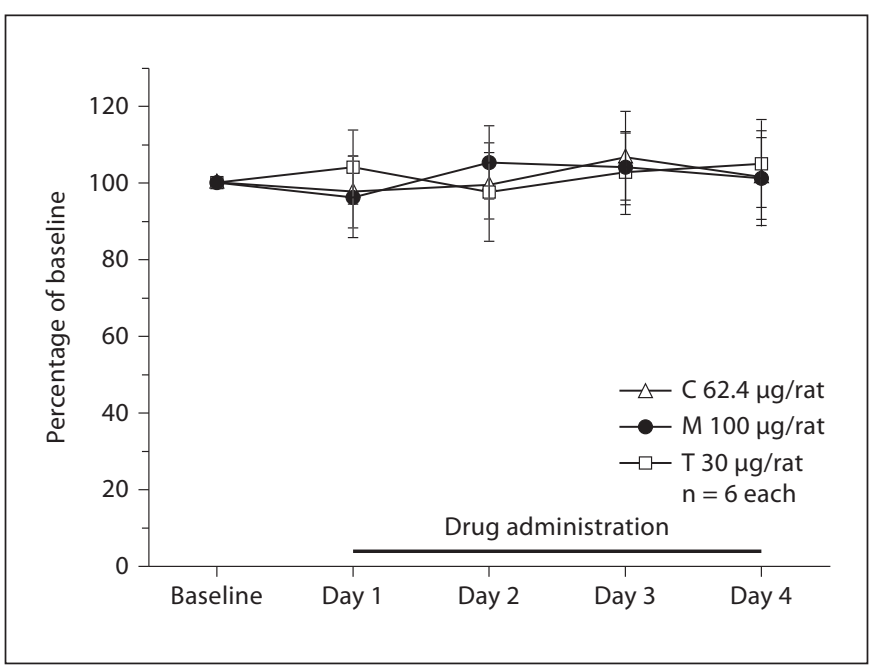

Fig. 7. Effects of drugs on motor performance of rats in the rotarod test. After a baseline response had been obtained, tramadol $30 \mu \mathrm{g}$, minocycline $100 \mu \mathrm{g}$, and combination $62.4 \mu \mathrm{g}$ were administered intrathecally and a rotarod test was performed once a day for 4 days. Compared with performance at baseline, there were no statistical differences from the rotarod test after intrathecal drug administration. Six rats in each group.

pendent manner. Therefore, suppression of neuronal activation through inhibiting $\mu$-opioid receptor activity could be an underlying mechanism of intrathecal tramadol on SNL-induced neuropathic pain. Besides, it is reported that tramadol could inhibit serotonin and norepinephrine reuptake at the spinal level $[28,35]$. It is known that serotonin and norepinephrine are two important neurotransmitters in the descending inhibition system, which could exert analgesic effects at the spinal level [36, 37]. Therefore, blocking reuptake of these two neurotransmitters may be another antiallodynic mechanism of tramadol observed in the present study.

\section{Minocycline Performed as a Glial Activation Inhibitor} when Treating Neuropathic Pain

Cumulating studies have shown a key role for activated spinal microglia in the development of nerve injury-induced neuropathic pain, especially during the initiation stage $[15,38]$. Previous reports indicate that minocycline could generate antinociceptive effects on neuropathic pain induced by peripheral nerve injury, inflammation or spinal cord injury [11,39-41]. Moreover, a previous study confirmed that intrathecal minocycline was effective for the treatment of SNL-induced neuropathic pain even after the established pain model [42].
The therapeutic time window for intrathecal minocycline is the initiation period (no more than 1 week) of peripheral nerve injury-induced neuropathic pain. In accordance to previous reports $[13,39,40]$, the present work confirmed that intrathecal minocycline shows obvious antiallodynic effects on SNL-induced neuropathic pain. The study indicated that intrathecal minocycline could block carrageenan-induced hyperalgesia and inhibited microglial activation with attenuation of the increased p-p38 in microglia [40]. In addition, minocycline also suppressed lipopolysaccharide-evoked spinal microglial activation. These results suggested that intrathecal minocycline produces a potent and consistent antinociception in models of chronic pain, which is mediated by direct inhibition of spinal microglial activation. In the present experiment, intrathecal minocycline also attenuated SNL-induced spinal dorsal horn microglial activation dose-dependently. This result indicated that inhibition of SNL-induced spinal microglial activation by minocycline is useful to conquer neuropathic pain, which may be a complementary option to modern neuronal-based therapeutics.

\section{Drug Combination Exhibited Synergistic Effects on}

Neuropathic Pain by Inhibiting Neuronal and

\section{Glial Activation Bilaterally}

The clinical concept of a balanced or associative manner proposed to use a combination of analgesics and other treatments to provide better pain relief and minimized side effects [5, 43]. However, most of the modern analgesic methods just focus on neuronal participation but neglect glial participation in the neuropathic pain progress. Since spinal glial cells play key roles in the initiation and maintenance of SNL-induced neuropathic pain $[1,15$, 44], combining a neuronal inhibitor with a glial inhibitor may propose a brand-new strategy for treating neuropathic pain more effectively.

The present study indicated that combination of tramadol with minocycline generates some synergistic antiallodynic effects on SNL-induced neuropathic pain in a dose-dependent manner. The antiallodynic effect of coadministration appeared stronger than that of intrathecal tramadol or minocycline alone. The experimental ED50 of the drug combination was much lower than that of the theoretical ED50. Moreover, the present report showed that combined tramadol $3.9 \mu \mathrm{g}$ with minocycline $27.3 \mu \mathrm{g}$ enhanced the depressing effects on SNL-induced spinal Fos expression and microglial activation, compared with that of intrathecal tramadol $10 \mu \mathrm{g}$ or minocycline $30 \mu \mathrm{g}$ individually. These results suggested that 
combination of tramadol with minocycline was effective to relieve SNL-induced mechanical allodynia by inhibition of neuronal and glial participation bilaterally.

The mechanisms underlying neuropathic pain are complicated. Evidence is available concerning the plasticity that occurs during the neuropathic pain progress. The most important contribution to plasticity during the initiation and the maintenance of neuropathic pain is neuron-glia interaction.

Peripheral nerve injury-induced spinal neuronal activation is followed by synthesis of various transmitters, excitatory amino acids, ATP, and chemokines such as fractalkine, which plays as the initiator of microglial activation. Fractalkine, also known as CX3CL1, was suggested to mediate signals between neuron and microglia. Fractalkine is synthesized and released by spinal neurons, whereas the sole receptor of fractalkine, CX3CR1, is expressed in spinal microglia after peripheral nerve injury [9]. This neuron-to-microglia interaction contributes to the development of nerve injury-induced neuropathic pain. Besides, ATP is actively released from injured primary afferents and dorsal horn neurons, which induce microglial activation after binding to the microglia P2X4 receptor [10]. Another study indicated that SNL induced spinal dorsal horn microgliosis, which directly contributed to the development of neuropathic pain [45]. Microglia expressed the neuregulin 1 receptor (NRG1R), which was a growth and differentiation factor. NRG1 stimulated microglial proliferation, chemotaxis, and survival, as well as interleukin- $1 \beta$ release in vitro via NRG1R. Intrathecal treatment with NRG1 resulted in microglial activation in the spinal dorsal horn. This microglial response was associated with the development of mechanical allodynia. Both an increase in NRG1 within the dorsal horn and activation of NRG1R specifically within microglia were observed after SNL. Blockade of the NRG1R or sequestration of endogenous NRG after SNL reduced the proliferation, the number of microglia with an activated morphology, and the expression of phospho-p38 by microglia. Furthermore, consequent to such changes, the mechanical allodynia was reduced [45]. Therefore, NRG1R signaling represents a novel pathway regulating the injury response of microglia. Additionally, neurotransmitters, neuromodulators, and inflammatory mediators, such as glutamate, ATP, substance P, CGRP, BDNF, IL-6, and CCL2, are released from primary afferent terminals into the spinal cord after nerve injury $[1,45$, 46]. These factors act on receptors on the postsynaptic nerve terminal, microglia, and astrocytes to modulate glial activity. Accordingly, the present study showed that intrathecal tramadol could suppress microglial activation with inhibition of SNL-induced OX42 upregulation. Furthermore, combination of tramadol with minocycline could synergistically relieve SNL-induced neuropathic pain with apparent suppression of microglial activation.

On the other hand, activated microglia released substances that influenced neuronal activity. SNL induced a quick response of spinal microglial activation, which appeared apparently 3 days after injury [38]. Activated microglia showed a stereotypic, progressive series of changes in morphology, gene expression, function and release of various chemical mediators, including proinflammatory cytokines (IL-1 $\beta$, IL-6, and TNF $\alpha$ ), complement components (C1q, C3, C4, C5, and C5a), and other substances that facilitate pain transmission, which might be involved in the development of neuropathic pain by modulating dorsal horn neuron activity [8, 47]. A study showed that IL-1 $\beta$ release from activated microglia was a response to the neuronal activation [48]. Thus, suppressing microglial activation could inhibit neuronal activation. Moreover, microglial activation can lead to opioid system alteration, which is recognized as resistance to morphine when treating neuropathic pain [47]. Another study indicated that suppressing microglial activation with minocycline enhanced morphine-induced analgesia [25]. Therefore, pharmacological combination of minocycline (microglial activation inhibitor) with tramadol ( $\mu$-opioid receptor agonist) may not only propose a potential strategy for treating neuropathic pain but also enhance the efficacy of tramadol. According to this concept, the present study indicated that combined minocycline with tramadol synergistically relieved SNL-induced neuropathic pain and downregulated spinal Fos activation.

Besides, neuron-microglia-astrocyte interactions have already been verified during the development of neuropathic pain $[8,46,49]$. However, previous studies also confirmed that microglia were mainly responsible for the initiation while astrocytes were mainly responsible for the maintenance of neuropathic pain $[15,49]$. Moreover, the present study was designed to detect the effects of drug application during the initiation stage of nerve injury-induced neuropathic pain. Therefore, this study did pay more attention to microglia participation. However, astrocyte participation cannot be neglected and research on this issue will consummate our knowledge of neuronmicroglia-astrocyte interactions.

The present results suggested that intrathecal injection of the neuronal activity inhibitor tramadol, or of the 
microglial activation modulator minocycline, could attenuate peripheral nerve injury-induced neuropathic pain effectively. Moreover, combination of tramadol with minocycline could exert some synergistic effects on neuropathic pain with apparent inhibition of neuronal and microglial activity. In accordance with a recent report [1] concerning the modern concept of the spinal mechanisms of neuropathic pain development, our previous report has shown that there is a positive feedback loop between neuronal and glial activation [23]. Therefore, the present study may propose a new strategy for treating peripheral nerve injury-induced neuropathic pain by breaking the bilateral interaction between neuron and glia.

\section{Acknowledgements}

This work was supported by grants from the National Natural Science Foundation of China (30901400, 81270016, 81100732). Innovation Research Team Program of Ministry of Education of China (IRT0560), and National Program of Basic Research of China (G2006CB500808).

\section{Disclosure Statement}

The authors declare that they have no competing interests.

\section{References}

1 Gao YJ, Ji RR: Chemokines, neuronal-glial interactions, and central processing of neuropathic pain. Pharmacol Ther 2010;126:5668.

-2 Guo W, Wang H, Watanabe M, Shimizu K, Zou S, LaGraize SC, Wei F, Dubner R, Ren K: Glial-cytokine-neuronal interactions underlying the mechanisms of persistent pain. J Neurosci 2007;27:6006-6018.

3 Wrzosek A, Obara I, Wordliczek J, Przewlocka B: Efficacy of tramadol in combination with doxepin or venlafaxine in inhibition of nociceptive process in the rat model of neuropathic pain: an isobolographic analysis. J Physiol Pharmacol 2009;60:71-78.

4 Kraychete DC, Sakata RK, Issy AM, Bacellar O, Jesus RS, Carvalho EM: Proinflammatory cytokines in patients with neuropathic pain treated with tramadol. Rev Bras Anestesiol 2009;59:297-303.

5 Alvarez P, Saavedra G, Hernandez A, Paeile C, Pelissier T: Synergistic antinociceptive effects of ketamine and morphine in the orofacial capsaicin test in the rat. Anesthesiology 2003;99:969-975.

-6 Guneli E, Karabay Yavasoglu NU, Apaydin S, Uyar M: Analysis of the antinociceptive effect of systemic administration of tramadol and dexmedetomidine combination on rat models of acute and neuropathic pain. Pharmacol Biochem Behav 2007;88:9-17.

7 Tsuda M, Inoue K, Salter MW: Neuropathic pain and spinal microglia: a big problem from molecules in 'small' glia. Trends Neurosci 2005;28:101-107.

8 Scholz J, Woolf CJ: The neuropathic pain triad: neurons, immune cells and glia. Nat Neurosci 2007;10:1361-1368.

-9 Milligan ED, Sloane EM, Watkins LR: Glia in 17 pathological pain: a role for fractalkine. J Neuroimmunol 2008;198:113-120.
10 Ikeda H, Tsuda M, Inoue K, Murase K: Longterm potentiation of neuronal excitation by neuron-glia interactions in the rat spinal dorsal horn. Eur J Neurosci 2007;25:1297-1306.

11 Guasti L, Richardson D, Jhaveri M, Eldeeb K, Barrett D, Elphick MR, Alexander SP, Kendall D, Michael GJ, Chapman V: Minocycline treatment inhibits microglial activation and alters spinal levels of endocannabinoids in a rat model of neuropathic pain. Mol Pain 2009;5:35.

$\checkmark 12$ Cui Y, Liao XX, Liu W, Guo RX, Wu ZZ, Zhao CM, Chen PX, Feng JQ: A novel role of minocycline: attenuating morphine antinociceptive tolerance by inhibition of p38 MAPK in the activated spinal microglia. Brain Behav Immun 2008;22:114-123.

13 Lin CS, Tsaur ML, Chen CC, Wang TY, Lin CF, Lai YL, Hsu TC, Pan YY, Yang CH, Cheng JK: Chronic intrathecal infusion of minocycline prevents the development of spinal-nerve ligation-induced pain in rats. Reg Anesth Pain Med 2007;32:209-216.

14 Coull JA, Beggs S, Boudreau D, Boivin D, Tsuda M, Inoue K, Gravel C, Salter MW, De Koninck Y: BDNF from microglia causes the shift in neuronal anion gradient underlying neuropathic pain. Nature 2005;438:10171021.

15 Zhuang ZY, Gerner P, Woolf CJ, Ji RR: ERK is sequentially activated in neurons, microglia, and astrocytes by spinal nerve ligation and contributes to mechanical allodynia in this neuropathic pain model. Pain 2005; 114: 149-159.

16 Ren K, Dubner R: Neuron-glia crosstalk gets serious: role in pain hypersensitivity. Curr Opin Anaesthesiol 2008;21:570-579.

Zimmermann M: Ethical guidelines for investigations of experimental pain in conscious animals. Pain 1983;16:109-110.
18 Kim SH, Chung JM: An experimental model for peripheral neuropathy produced by segmental spinal nerve ligation in the rat. Pain 1992;50:355-363.

19 Milligan ED, Twining C, Chacur M, Biedenkapp J, O'Connor K, Poole S, Tracey K, Martin D, Maier SF, Watkins LR: Spinal glia and proinflammatory cy tokines mediate mirrorimage neuropathic pain in rats. J Neurosci 2003;23:1026-1040.

20 Chaplan SR, Bach FW, Pogrel JW, Chung JM, Yaksh TL: Quantitative assessment of tactile allodynia in the rat paw. J Neurosci Methods 1994;53:55-63.

21 Arguelles CF, Torres-Lopez JE, GranadosSoto V: Peripheral antinociceptive action of morphine and the synergistic interaction with lamotrigine. Anesthesiology 2002;96: 921-925.

22 Tallarida RJ, Porreca F, Cowan A: Statistical analysis of drug-drug and site-site interactions with isobolograms. Life Sci 1989;45: 947-961.

23 Wang W, Wang W, Mei X, Huang J, Wei Y, Wang Y, Wu S, Li Y: Crosstalk between spinal astrocytes and neurons in nerve injuryinduced neuropathic pain. PLoS One 2009; 4:e6973.

24 Leventhal L, Smith V, Hornby G, Andree TH, Brandt MR, Rogers KE: Differential and synergistic effects of selective norepinephrine and serotonin reuptake inhibitors in rodent models of pain. J Pharmacol Exp Ther 2007;320:1178-1185.

-25 Hutchinson MR, Northcutt AL, Chao LW, Kearney JJ, Zhang Y, Berkelhammer DL, Loram LC, Rozeske RR, Bland ST, Maier SF, Gleeson TT, Watkins LR: Minocycline suppresses morphine-induced respiratory depression, suppresses morphine-induced reward, and enhances systemic morphine-induced analgesia. Brain Behav Immun 2008; 22:1248-1256.
Tramadol and Minocycline for Neuropathic Pain 
26 Chakraborty S, Chakrabarti J, Bhattacharya D: Intrathecal tramadol added to bupivacaine as spinal anesthetic increases analgesic effect of the spinal blockade after major gynecological surgeries. Indian J Pharmacol 2008;40:180-182.

27 Jou IM, Chu KS, Chen HH, Chang PJ, Tsai YC: The effects of intrathecal tramadol on spinal somatosensory-evoked potentials and motor-evoked responses in rats. Anesth Analg 2003;96:783-788.

28 Wilder-Smith $\mathrm{CH}$, Schimke J, Osterwalder B, Senn HJ: Oral tramadol, a mu-opioid agonist and monoamine reuptake-blocker, and morphine for strong cancer-related pain. Ann Oncol 1994;5:141-146.

-29 Norrbrink C, Lundeberg T: Tramadol in neuropathic pain after spinal cord injury: a randomized, double-blind, placebo-controlled trial. Clin J Pain 2009;25:177-184.

30 Erichsen HK, Hao JX, Xu XJ, BlackburnMunro G: Comparative actions of the opioid analgesics morphine, methadone and codeine in rat models of peripheral and central neuropathic pain. Pain 2005;116:347-358.

31 Hammond DL, Wang H, Nakashima N, Basbaum AI: Differential effects of intrathecally administered delta and mu opioid receptor agonists on formalin-evoked nociception and on the expression of Fos-like immunoreactivity in the spinal cord of the rat. J Pharmacol Exp Ther 1998;284:378-387.

-32 Bullitt E: Expression of c-Fos-like protein as a marker for neuronal activity following noxious stimulation in the rat. J Comp Neurol 1990;296:517-530.

- 33 Dai Y, Kondo E, Fukuoka T, Tokunaga A, Miki K, Noguchi K: The effect of electroacupuncture on pain behaviors and noxious stimulus-evoked Fos expression in a rat model of neuropathic pain. J Pain 2001;2: 151-159.
34 Li C, Sekiyama H, Hayashida M, Takeda K, Sumida T, Sawamura S, Yamada Y, Arita H, Hanaoka K: Effects of topical application of clonidine cream on pain behaviors and spinal Fos protein expression in rat models of neuropathic pain, postoperative pain, and inflammatory pain. Anesthesiology 2007; 107:486-494

35 Berrocoso E, De Benito MD, Mico JA: Role of serotonin 5-HT1A and opioid receptors in the antiallodynic effect of tramadol in the chronic constriction injury model of neuropathic pain in rats. Psychopharmacology (Berl) 2007;193:97-105.

- 36 Kino Y, Tanabe M, Honda M, Ono H: Involvement of supraspinal imidazoline receptors and descending monoaminergic pathways in tizanidine-induced inhibition of rat spinal reflexes. J Pharmacol Sci 2005;99:5260.

37 Iyengar S, Webster AA, Hemrick-Luecke SK, $\mathrm{Xu}$ JY, Simmons RM: Efficacy of duloxetine, a potent and balanced serotonin-norepinephrine reuptake inhibitor in persistent pain models in rats. J Pharmacol Exp Ther 2004;311:576-584.

38 Jin SX, Zhuang ZY, Woolf CJ, Ji RR: P38 mitogen-activated protein kinase is activated after a spinal nerve ligation in spinal cord microglia and dorsal root ganglion neurons and contributes to the generation of neuropathic pain. J Neurosci 2003;23:4017-4022.

39 Ledeboer A, Sloane EM, Milligan ED, Frank MG, Mahony JH, Maier SF, Watkins LR: Minocycline attenuates mechanical allodynia and proinflammatory cytokine expression in rat models of pain facilitation. Pain 2005; 115:71-83.

40 Hua XY, Svensson CI, Matsui T, Fitzsimmons B, Yaksh TL, Webb M: Intrathecal minocycline attenuates peripheral inflammation-induced hyperalgesia by inhibiting p38 MAPK in spinal microglia. Eur J Neurosci 2005;22:2431-2440.

-41 Mika J, Rojewska E, Makuch W, Przewlocka B: Minocycline reduces the injury-induced expression of prodynorphin and pronociceptin in the dorsal root ganglion in a rat model of neuropathic pain. Neuroscience 2010;165:1420-1428.
2 Mei XP, Xu H, Xie C, Ren J, Zhou Y, Zhang $\mathrm{H}, \mathrm{Xu}$ LX: Post-injury administration of minocycline: an effective treatment for nerveinjury induced neuropathic pain. Neurosci Res 2011;70:305-312.

43 Lynch ME, Clark AJ, Sawynok J, Sullivan MJ: Topical amitriptyline and ketamine in neuropathic pain syndromes: an open-label study. J Pain 2005;6:644-649.

44 Piao ZG, Cho IH, Park CK, Hong JP, Choi SY, Lee SJ, Lee S, Park K, Kim JS, Oh SB: Activation of glia and microglial p38 MAPK in medullary dorsal horn contributes to tactile hypersensitivity following trigeminal sensory nerve injury. Pain 2006;121:219-231.

45 Calvo M, Zhu N, Tsantoulas C, Ma Z, Grist J, Loeb JA, Bennett DL: Neuregulin-ERBB signaling promotes microglial proliferation and chemotaxis contributing to microgliosis and pain after peripheral nerve injury. J Neurosci 2010;30:5437-5450.

46 Milligan ED, Watkins LR: Pathological and protective roles of glia in chronic pain. Nat Rev Neurosci 2009;10:23-36.

47 Mika J: Modulation of microglia can attenuate neuropathic pain symptoms and enhance morphine effectiveness. Pharmacol Rep 2008;60:297-307.

48 Li Y, Liu L, Kang J, Sheng JG, Barger SW, Mrak RE, Griffin WS: Neuronal-glial interactions mediated by interleukin-1 enhance neuronal acetylcholinesterase activity and mRNA expression. J Neurosci 2000;20:149155.

49 Wang W, Mei XP, Wei YY, Zhang MM, Zhang T, Xu LX, Wu SX, Li YQ: Neuronal NR2B-containing NMDA receptor mediates spinal astrocytic c-Jun $\mathrm{N}$-terminal kinase activation in a rat model of neuropathic pain. Brain Behav Immun 2011;25:1355-1366. 